\title{
ABBREVIATED COURSE OF RADIATION THERAPY WITH CONCURRENT TEMOZOLOMIDE FOR HIGH-GRADE GLIOMA
}

\author{
Waleed El-Sayed Hamoda, M.D.; Yosra Taha Metwaly, M.D.; Nabila Hefzi Abd El-Hakem, \\ M.D.; and Eman Ali El-Sebai, MB.B.CH.
}

The Department of Clinical Oncology and Nuclear Medicine, Zagazig University, Egypt.

\begin{abstract}
Purpose: To evaluate efficacy and safety of hypofractionated radiotherapy concurrently with temozolamide in treatment of high grade gliomas.

Method: 54 patients with GBM enrolled and divided in two groups, the group A consists of 27 patients treated prospectively by three dimentional (3D) conformal radiotherapy at a dose of 40Gy / 15 fraction over 3 weeks and compared with the other group B which consists of 27 patients treated retrospectively at a dose of 60Gy / 30 fraction over 6 weeks, both groups received temozolamide concurrently with radiotherapy.

Results: Over all response was $59.3 \%$ in A group and 55.6\% in B group without significant statistical difference in both groups. The median follow up in both groups was 11 months. $6^{\text {th }}$ month PFS in group A was $58.6 \%$ and in group B $52.7 \%$ without statistical significant difference. $6^{\text {th }}$ month OS in group A was $81.5 \%$ and in group B $85.2 \%$ without statistical significant difference. On univariate analysis of the prognostic factors of progression the extent of surgery, histological subtype and response to radiotherapy are significant predictors. There is no significant statistical difference in toxicity of treatment between both groups.
\end{abstract}

Conclusion: HRT concurrently with temozolamide may be used as an option in treatment of high grade glioma.

Key Words: Conform al hypofractionated radiotherapy, temozolamide, gliomas.

\section{INTRODUCTION}

$\mathrm{H}$ igh grade gliomas are aggressive and frequent brain tumors that can occur at any age groups with high percentage in the $5^{\text {th }}$ and $6^{\text {th }}$ decades, their percentage was $35-45 \%$ of all brain tumors in adults ${ }^{[1]}$. The overall prognosis remains poor with high grade gliomas $^{[2]}$. GBM rapidly progresses, death may occur within 1 year of diagnosis. Symptoms affect seriously quality of life, so different treatment modality (surgery, radiation, chemotherapy and palliation) is chosen according to different patients ${ }^{[1]}$.

Despite of various aggressive treatment strategies, the prognosis is very poor with a median survival time of slightly more than 1 year ${ }^{[3]}$. Life expectancy after operation alone is about 4 months, messicating more effective treatment alternatives ${ }^{[4]}$.

Radiotherapy was the most effective treatment for these tumors, but RT alone can only prevent recurrence or progression ${ }^{[5]}$. Radiation therapy (RT) improves survival in patients with malignant gliomas as compared to supportive care alone ${ }^{[\mathbf{6}]}$.

Temozolomide (TMZ), an oral alkylating agent with good CNS penetrance has been shown to improve survival with minimal hematologic toxicity when given concurrently with conventionally fractionated radiation $(60$ Gy) ${ }^{[7]}$. Decreasing the overall treatment time and improving the general condition of patients should be achieve. Conventional RT treatment need 6-7 weeks ${ }^{[8]}$. Multiple studies have found that decreasing the course of RT may be a selected option for high grade glioma $^{[9]}$.

Because of the worse prognosis of patients with high grade glioma, efforts have been made to reduce the intensity and duration of the treatment for those patients in order to minimize potential toxic effects of the treatment and inconvenience associated with multiple clinic or hospital visits ${ }^{[10]}$.

Data from retrospective studies and prospective trials have shown that abbreviated 
course of radiation therapy concurrently with temozolamide delivering a slightly lower dose of radiation can achieve similar results in old patients or patients with poor performance status in comparison with the standard 6-7 week course of radiation therapy. Such shortened radiotherapy concurrently with temozolamide schedules would be beneficial for patients with limited life expectancy ${ }^{[11]}$.

\section{PATIENTS AND METHODS}

The study was conducted to 54 patients of newly diagnosed high grade glioma reffered to the Clinical Oncology \& Nuclear Medicine Department, Zagazig University Hospitals, from $2 / 2013$ to $6 / 2015$.

The patients was divided in two arms, Arm A include 27 patients treated prospectivly by hypofractionated radiotherapy at a dose 40Gy / 15 fractions and compared with Arm B that include 27 patients treated retrospectively by conventional radiotherapy at a dose 60Gy / 30 fractions. The patients included are those of newly diagnosed pathologically confirmed high grade glioma, age $>18$ years old, KPS $\geq 50$ and with no previous radiation or other cancer. Patients with recurrent glioma or low grade glioma were not included.

\section{Treatment Details}

Patients received adjuvant three dimentional conformal radiotherapy. Which means conforming the prescriped dose to the target volume while decreasing dose to the normal tissues through many steps. These steps including patient's immobilization, CT planning, target volume delineation and critical structures, beam design and shaping of field with multi leaf colimator, dose calculation, plane optimization, and verification. The radiotherapy started within 3-6 weeks after surgery a dose of $40 \mathrm{~Gy}$ in 15 fractions (2.6 Gy daily); given over 3 weeks in group (A) and a dose 60 Gy in 30 fractions (2 Gy daily) given over 6 weeks in group (B). Chemotherapy is given concurrently using temozolomide (TMZ) daily $75 \mathrm{mg} / \mathrm{m} 2$ during radiotherapy ( $\mathrm{RT}$ ).

\section{Surveillance and follow-up:}

Assessment was carried out weekly during radiotherapy by Physical, neurological examination, and assessment of performance status. A clinical assessment of neurological status and tolerance to treatment was done monthly. Imaging was done six to eight weeks after radiotherapy and at three months intervals in subsequent follow up.

\section{STATISTICAL ANALYSIS}

All data analyzed by using SPSS 18.0. Continuous variables expressed as the mean \pm SD \& median (range), and the categorical variables were expressed as a number (percentage). Continuous variables checked for tested by using Shapiro-Wilk test. Independent sample Student-t test was used to compare two groups of data with normal distribution while Mann Whitney U test was used for data with non-normal distribution. Percent of categorical variables compared using the Chi-square $\left(\chi^{2}\right)$ test. Multivariate regression model created to define the independent predictors of response. In univariate analysis variables with a $\mathrm{p}$-value $<0.20$ are included in the multivariate model. OS is defined as the time from surgery to death or last known alive (censored). PFS was defined from time of achievement of a certain response to the date of progression. Stratification of OS, \& PFS was done according to all possible prognostic factors. These event-to-time distributions estimated using Kaplan-Meier test, and compared using two-sided exact stratified log-rank tests. The Cox proportional hazards regression model was used to detect the independent prognostic factors as well as to detect their effects on PFS \& OS. In univariate Cox regression analysis variables give a $p$ value $<0.20$ included in the multivariate Cox regression model. All tests were two sided with $\mathrm{p}<0.05$ was considered significant (S), and $\mathrm{p}>0.05$ considered non significant (NS) statistically.

\section{RESULTS}

The current study include 54 patients of high grade glioma divided in two groups, Arm A include 27 patients treated prospectivly by hypofractionated radiotherapy at a dose 40Gy / 15 fractions and compared with Arm B that include 27 patients treated retrospectively by conventional radiotherapy at a dose 60Gy / 30 fractions, patients characterstics (Table 1). 
Table (1): Basic characteristics.

\section{Basic characteristics}

\begin{tabular}{|c|c|c|c|c|}
\hline Age & $58(43-66)$ & $53(28-63)$ & $1.947^{t}$ & 0.052 \\
\hline \multicolumn{5}{|l|}{ Sex } \\
\hline Male & $7(25.9 \%)$ & $9(33.3 \%)$ & $0.078 *$ & 0.780 \\
\hline Female & $20(74.1 \%)$ & $18(66.7 \%)$ & & \\
\hline $50-70 \%$ & $16(59.3 \%)$ & $19(70.4 \%)$ & $0.731 *$ & 0.393 \\
\hline$>70 \%$ & $11(40.7 \%)$ & $8(29.6 \%)$ & & \\
\hline Frontal & $5(18.5 \%)$ & $4(14.8 \%)$ & $0.133^{*}$ & 0.715 \\
\hline Parietal & $9(33.3 \%)$ & $8(29.6 \%)$ & $0.086^{*}$ & 0.770 \\
\hline Temporal & $1(3.7 \%)$ & $0(0 \%)$ & $1.019 *$ & 0.313 \\
\hline Occipital & $2(7.4 \%)$ & $0(0 \%)$ & $2.077 *$ & 0.150 \\
\hline Fronto-parietal & $3(11.1 \%)$ & $9(33.3 \%)$ & $3.857 *$ & 0.050 \\
\hline Parieto-occipital & $1(3.7 \%)$ & $1(3.7 \%)$ & $0.000 *$ & 1.000 \\
\hline Tempro-parietal & $6(22.3 \%)$ & $5(18.5 \%$ & $0.114 *$ & 0.735 \\
\hline Biopsy & $6(22.2 \%)$ & $10(37 \%)$ & $1.421 *$ & 0.233 \\
\hline STE & $11(40.7 \%)$ & $10(37 \%)$ & $0.078 *$ & 0.780 \\
\hline GTE & $10(37 \%)$ & $7(25.9 \%)$ & $0.773 *$ & 0.379 \\
\hline Anaplastic astrocytoma & $8(29.6 \%)$ & $7(25.9 \%)$ & $0.092 *$ & 0.761 \\
\hline Glioblastoma multiform (GBM) & $19(70.7 \%)$ & $20(74.1 \%)$ & & \\
\hline
\end{tabular}

Continuous variables were expressed as the median (range); Categorical variables were expressed as number (percentage); + Mann Whitney $U$ test;* Chi-square test; $p<0.05$ is significant.

- Median age in group A was 58 years old and in group B 53 years old. Male represent 59.3\% in A group and $63 \%$ in B group, while female represent $40.7 \%$ in A group and $37 \%$ in B group.

- KPS $>70 \%$ in $40.7 \%$ of patients in A group and $29.6 \%$ of patients in B group.

- The majority of patients represented by headache about $63 \%$ of patients in A group and $51.9 \%$ of patients in B group, while the rest of patients represented by convulsion, motor weakness and gait disturbance.

- Most patients underwent surgery 37\% in A group and 25.9\% in B group underwent GTE, while $40.7 \%$ in A group and 37\% in B group underwent STE. Biopsy was performed in $22.2 \%$ in group A and $37 \%$ in group B.

- Anaplastic astrocytoma found in 8 patients in group A, 7 patients in group B, while GBM found in 19 patients in group A, 20 patients in group B.

After treatment the radiological response was assessed (Table 2). 
Table (2): Radiological response, recurrence and mortality.

\begin{tabular}{|c|c|c|c|c|}
\hline No response (NR) & $11(40.7 \%)$ & $12(44 \%)$ & $0.076^{*}$ & 0.783 \\
\hline Response (OAR) & $16(59.3 \%)$ & $15(55.6 \%)$ & & \\
\hline PD & $6(22.2 \%)$ & $7(25.9 \%)$ & $0.277 *$ & 0.964 \\
\hline$\overline{\mathrm{SD}}$ & $5(18.5 \%)$ & $5(18.5 \%)$ & & \\
\hline$\overline{\mathrm{PR}}$ & $13(48.15 \%)$ & $13(48 \%)$ & & \\
\hline $\mathrm{CR}$ & $3(11.1 \%)$ & $2(7 \%)$ & & \\
\hline Progression & $13(48.1 \%)$ & $12(44.4 \%)$ & $1.944^{*}$ & 0.857 \\
\hline Mortality & $13(48.1 \%)$ & $12(44.4 \%)$ & $1.944^{*}$ & 0.857 \\
\hline Median PFS & 12 months & $\mathrm{NR}$ & $0.013 t$ & 0.909 \\
\hline 6 months PFS & $58.6 \%$ & $52.7 \%$ & & \\
\hline 12 months PFS & $45.4 \%$ & $52.7 \%$ & & \\
\hline HR $(95 \% \mathrm{CI})$ & & & & \\
\hline
\end{tabular}

\begin{tabular}{lcccc}
\hline Median OS & 16 months & NR & $0.108 \ddagger$ & 0.741 \\
\hline 6 months OS & $81.5 \%$ & $85.2 \%$ & & \\
\hline 12 months OS & $53.7 \%$ & $51 \%$ & & \\
\hline 18 months OS & $47 \%$ & $51 \%$ & & \\
\hline
\end{tabular}

HR $(95 \% \mathrm{CI})$

Categorical variables were expressed as number (percentage); Chi-square test; $¥$ Logrank test; NR: not reached yet; HR: Hazard ratio; 95\%CI: 95\% confidence interval; $p<0.05$ is significant.

- Over all response was 59.3\% in A group and 55.6\% in B group without significant statistical difference.

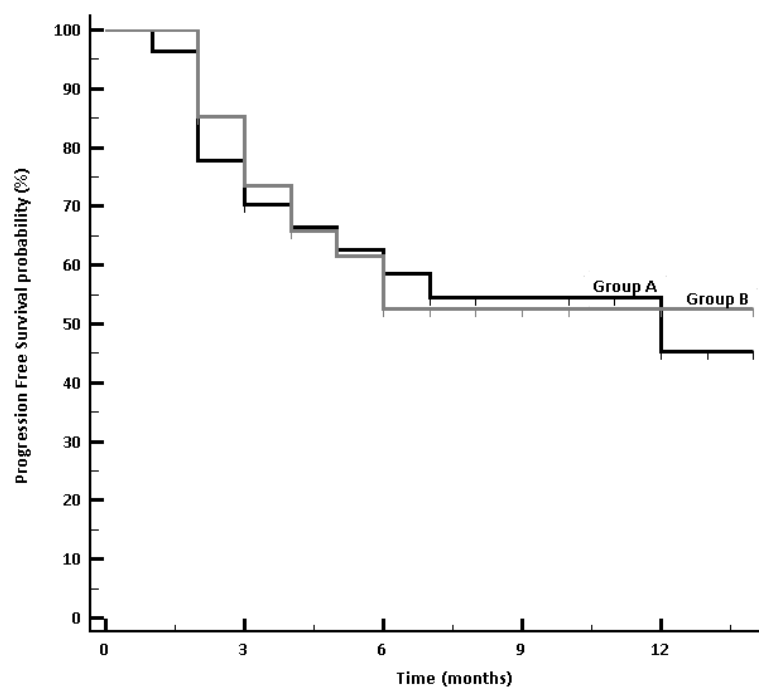

(A)

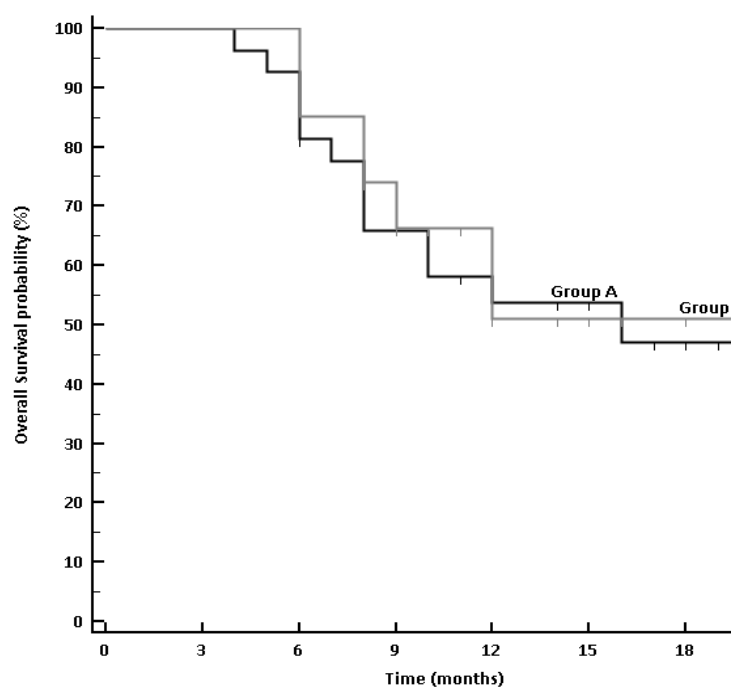

(B)

Fig. (1): Kaplan Meier plot of survival in relation to treatment arms. (A) progression free survival (B) Overall survival. 
The $6^{\text {th }}$ month PFS in group A was $58.6 \%$ and in group B $52.7 \%$ without statistical significant difference. $6^{\text {th }}$ month OS in group A was $81.5 \%$ and in group B $85.2 \%$ without statistical significant difference. On univariate analysis of the prognostic factors of progression the extent of surgery, histological subtypes and response to radiotherapy were significant predictors.

On univariate analysis of potential predictors for mortality extent of surgery, histopathological subtypes, response to radiotherapy and progression were significant predictors.

\section{DISCUSSION}

The treatment of GBM represent a serious problem clinically. If adjuvant treatment is not given after surgical interference, the median survival will be affected seriously and may be about 4 months ${ }^{(\mathbf{1 2})}$. Surgery and adjuvant radiation are the standard treatment $^{(\mathbf{1 3})}$. The dose of radiation used in different randomized studies is 60Gy/2Gy per fraction over 6 weeks ${ }^{(14)}$. Adjuvant hypofractionated radiation had several advantages over the convential radiation including mainly radiobiological advantage of hypofractionation with glioma as a neural tissue which is a late responding tissue with low Alpha/Beta that responeding better with higher dose per fraction, a cost advantage over the conventional radiation resulting from fewer number of treatment session sparing hospital resources and finally shorter overall treatment time had an important concern in the quality of life of the patients reducing their hospital visits ${ }^{(15)}$.

In our study evaluating the effecacy and toxicity of hypofractionated radiotherapy in treatment of high grade glioma, we concluded that there is no significant statistical difference in survival between hypofractionated and conventional radiation with the 6 and 12 months OS rates were $81.5 \%$ and $53.7 \%$ in group $\mathrm{A}$ (hypofrationated arm) and $85.2 \%$ and $51 \%$ in group B (conventional arm) respectively. The 6 and 12 months PFS rates were $58.6 \%$ and $45.4 \%$ in group A (hypofrationated arm) and $52.7 \%$ and $52.7 \%$ in group B (conventional arm) respectively. Over all response was
$59.3 \%$ in A group and $55.6 \%$ in B group without significant statistical difference. On univariate analysis of the prognostic factors of progression extent of surgery, histopathological subtype and response to radiotherapy were significant predictors.

Slotman et al. ${ }^{(16)}$ treated 30 patients with hypofractionated radiotherapy using 42Gy over 3 weeks. The median follow up time was two years. $80 \%$ of patients suffered from recurrence after 6.5 months. Age PS \& extent of surgery were found as prognostic factors. The study detect that hypofractionated radiotherapy were similar to normal fractionated radiotherapy. Acute or late complications not detected.

Glinski et al. ${ }^{(17)}$ treated 44 patients by conventional radiotherapy or HRT in their trial. Survival at 2 year was $23 \%$ in the HRT arm and $10 \%$ in the conventional arm. Treatment was tolerable in both arms.

McAleese et al. ${ }^{(\mathbf{1 8})}$ had compared 2 groups of bad prognosis GBM patients one group treated by HRT 5 Gy/fraction 2 times per week up to $30 \mathrm{~Gy}$ the other group was treated by normal fractionated RTH and found that HRT could be a reasonable with lesser survival benefit thanconventional RTH.

In the randomized trial done at sites in 7 European countries, 342 patients with GBM were included. Patients were treated by conventional $60 \mathrm{~Gy} / 2 \mathrm{~Gy}$ per fractions over 6 weeks ( $\mathrm{n}=100$ ), hypofractionated $34 \mathrm{~Gy} / 3,4 \mathrm{~Gy}$ per fractions over 2 weeks $(n=123)$, or $200 \mathrm{mg} / \mathrm{m} 2$ temozolomide daily on days 1 to 5 every 4 weeks for 6 cycles $(n=119)$. Median survival was 7.5 months for hypofractionated radiation $34 \mathrm{~Gy}, 8.3$ months for temozolomide and 6 months for conventional radiation. There was no significant survival benefite for patients treated with hypofractionated radiation or temozolomide. These results concluded that HRT or temozolomide could replace the conventional radiation in patients with glioblastoma ${ }^{(\mathbf{1 9})}$.

Abdelgawad et al. ${ }^{(\mathbf{2 0})}$ compared conventional radiotherapy with hypofractionated radiotherapy in treatment of GBM and concluded that there is no difference in the survival was detected with slightly better survival in HRT group but of no statistical 
significance. Progression free survival and overall survival were 6 and 5 months, and 6.5 and 6 months for hypofractionated radiotherapy and conventional radiotherapy groups respectively.

Toson et al. ${ }^{\text {(21) }}$ all of 30 patients with median age 73 years received adjuvant hypofractionated radiotherapy at a dose of 45Gy/3Gy per fraction over three weeks. Concurrent chemotherapy consists of temozolamide (TMZ) at a dose of $75 \mathrm{mg} / \mathrm{m} 2$ daily followed by 6 cycles of adjuvant TMZ at a dose of $200 \mathrm{mg} / \mathrm{m} 2$ for 5 days every 28 days. Four (13.33\%) patients achieved complete response (CR) and 5 (16.66\%) patients had partial response (PR). The median overall survival and PFS were 11 and 10 months respectively. The median followup time was 20 months (range 4-42). On univariat analysis of the prognostic factors, the type surgery and performance status were found to have significant effect on the survival ( $\mathrm{p}=0.002$ and 0.001 respectively). On multivariate cox regression analysis, performance status was the only significant prognostic independent factor $(\mathrm{p}=0.005)$.

\section{CONCLUSION AND RECOMMENDATION}

HRT concurrently with temozolamide may be used as an option in treatment of high grade glioma patients. However, future researches are needed to detect the optimal hypofractionated schedule and dose.

\section{REFRENCES}

1. Meral Y, Sayin Bektas Kaya Bekir H, Bakkal Kadri Altundag Muzaffer B, et al. The results of hypofractionated radiotherapy in 31 patients with high-grade gliomas. Med Oncol, 2007; 24: 379-383.

2. Mizuhiko T, Tomoko E, Shinji N, et al. A pilot study of hypofractionated radiation therapy with temozolomide for adults with glioblastoma multiforme. J Neurooncol, 2011; 102: 247-253.

3. Stupp R, Hegi ME, Mason WP, van den Bent MJ et al. Effects of radiotherapy with concomitant and 7adjuvant temozolomide versus radiotherapy alone on survival in glioblastoma in a randomised phase III study: 5-year analysis of the EORTC-NCIC trial. Lancet Oncol, 2009; 10: 459-466.

4. Simpson JR et al. Influence of location and extent of surgical resection on survival of patients with glioblastoma multiforme: results of three consecutive Radiation Therapy Oncology Group (RTOG) clinical trials. Int J Radiat Oncol Biol Phys, 1993; 26:239-244.

5. Floyd NS et al. Hypofractionated intensitymodulated radiotherapy for primary glioblastoma multiforme. Int $\mathbf{J}$ Radiat Oncol Biol Phys, 2004; 58:721-726.

6. Keime-Guibert F, Chinot O, Taillandier L, et al. Radiotherapy for glioblastoma in the elderly. N Engl J Med, 2007; 356:1527-1535.

7. Curschmann J, Janzer RC, Ludwin SK, et al. Radiotherapy plus concomitant and adjuvant temozolomide for glioblastoma. N Engl J Med, 2005; 352:987-996.

8. Halperin EC Malignant gliomas in older adults with poor prog nostic signs. Getting nowhere, and taking a long time to do it. Oncology, 1995; 9:229-234.

9. Chang EL et al. Hypofractionated radiotherapy for elderly or younger with lowperformance status glioblastoma patients: outcome and prognostic factors. Int $\mathbf{J}$ Radiat Oncol Biol Phys, 2003; 56:519-528.

10.Sultanem K, et al. The use of hypofractionated intensity-modulated irradiation in the treatment of glioblastoma multiforme: preliminary results of a prospective trial. Int $\mathbf{J}$ Radiat Oncol Biol Phys, 2004; 58:247-252.

11.Simon S. Lo, and Eric L. Chang, Hypofractionated Radiation Therapy for Glioblastoma. American Society of Clinical Oncology, 2011; 1092-9118/10/1-10

12.Saleman M Glioblastoma multiforme and anaplatic astrocytoma. In: Kaye AH, Laws ER(eds): Brain Tumors: An encyclopedic approach Lnhurchill Livingstone, 2001; PP 493-523.

13.Laperrieve N, Zuraw L, Cairncross G, et al. Cancer Care Ontario Practice Guidelines Initiative Neuro-Oncology Disease Site Group. Radiotherapy for newly diagnosed malignant glioma in adults: a systematic review. Radiother Oncol, 2002; 64: 259-273.

14.MRC Brain Tumor Working Party A medical research council trial astrocytoma. $\mathrm{Br}$ J Cancer, 1991; 64(4): 769-774.

15.Arslan AN, Karadeniz G, Guveli MF, Postoperative hypofractionated radiotherapy in glioblastoma multiforme. Journal of BUON, 2006; 11: 39-42.

16.Slotman BJ, Kralendonk JH, van Alphen HAM et al. Hypofractionated radiation therapy in patients with glioblastome multiform: Results of treatment and impact of prognostic factors. Int $\mathrm{J}$ Radiat Oncal Bid Phys, 1996; 34: 895-898. 
17.Glinski B, Postoperative hypofractionated radiotherapy versus conventionally fractionated radiotherapy in malignant gliomas: A preliminary report on a randomized trial J Neuro Oncol, 1993; 16: 167-172.

18.McALeese JJ, Stenning SP, Ashley S et al. Hypofractionated radiotherapy for poor prognosis malignant glioma: matched pair survival analysis with MRC controls. Radiotherapy \& Oncology, 2003; 67: 177-182.

19.A Malmstrom BH, Grønberg R, Stupp C, et al. Glioblastoma (GBM) in elderly patients: A randomized phase III trial comparing survival in patients treated with 6-week radiotherapy (RT) versus hypofractionated RT over 2 weeks versus temozolomide single-agent chemotherapy (TMZ) J Clin Oncol, 2010; 28: 18s, (suppl; abstr LBA2002).

20.Abdelgawad $M$, Ismail E, Nawar $\mathbf{N}$, Hypofractionated radiotherapy versus conventional radiotherapy in treatment of glioblastoma multiform. Pan Arab Journal of Oncology, 2011; 4: 48-52

21.Toson EA, Sharaf El-Deen DA, El-Zafarany ME et al. Conformal Hypofractionated Radiotherapy Concomitant with and Followed By Temozolomide in High Grade Glioma in Elderly Patients. Med. J. Cairo Univ, 2012; Vol. 80, No. 1, 799-805. 